\title{
Klinefelter syndrome: an unusual diagnosis in pediatric patients
}

\author{
Síndrome de Klinefelter: diagnóstico raro na faixa etária pediátrica
}

\author{
Bruna J. Tincani,1,2, Bruno R. Mascagni ${ }^{3}$, Roberto D. P. Pinto ${ }^{3}$, Guilherme Guaragna-Filho ${ }^{1,2}$, \\ Carla C. T. S. Castro ${ }^{1,2}$, Letícia E. Sewaybricker ${ }^{1,2}$, Nilma L. Viguetti-Campos ${ }^{2,4}$, \\ Antonia P. Marques-de-Faria 2,5, Andréa T. Maciel-Guerra2,6, Gil Guerra-Júnior 2,7
}

\section{Resumo}

Objetivo: Identificar dados clínicos e laboratoriais que diferenciam os casos com síndrome de Klinefelter de acordo com a faixa etária.

Casuística e métodos: Foram incluídos todos os casos de hipogonadismo, ginecomastia e/ou infertilidade avaliados em hospital universitário cujo cariótipo foi realizado entre janeiro de 1989 e dezembro de 2011, totalizando 105 pacientes. Foram avaliados: idade na primeira consulta, relação entre envergadura e altura, pilificação pubiana, ginecomastia, tamanho testicular, hormônio luteinizante (LH), hormônio folículoestimulante (FSH), testosterona e espermograma.

Resultados: Foram diagnosticados três casos com síndrome de Klinefelter (SK+) e 72 sem a síndrome (SK-). Dos casos com síndrome de Klinefelter, apenas sete $(21,2 \%)$ foram diagnosticados antes dos 20 anos e dois $(6,1 \%)$ antes dos 10 anos de idade. A idade na primeira consulta (em anos) foi semelhante nos dois grupos (SK+ $=31,3 \pm 12,9$ e SK- $=27,6 \pm 12,1)$, o mesmo ocorrendo com a relação entre envergadura e altura e a presença de ginecomastia. No entanto, a pilificação pubiana foi menor no grupo SK+, o mesmo ocorrendo com a média do volume bitesticular e a testosterona, enquanto que o LH e o FSH foram mais elevados neste grupo, o mesmo ocorrendo com a frequência de azoospermia.

Conclusões: A síndrome de Klinefelter ainda é pouco e tardiamente diagnosticada em nosso meio, sendo os dados de tamanho testicular, $\mathrm{LH}, \mathrm{FSH}$, testosterona e presença de azoospermia no espermograma os mais importantes para o seu diagnóstico, principalmente na puberdade e na vida adulta.

J Pediatr (Rio J). 2012;88(4):323-7: Síndrome de Klinefelter, infertilidade masculina, puberdade tardia, ginecomastia.

\begin{abstract}
Objective: To identify clinical and laboratory data which differentiate Klinefelter syndrome (KS) patients according to age group.

Methods: The study included all cases of hypogonadism, gynecomastia and/or infertility whose karyotype was performed at a university hospital from January 1989 to December 2011, in a total of 105 subjects. The following data were retrospectively analyzed: age at first visit, ratio of arm span to height, pubic hair, gynecomastia, testicular volume, luteinizing hormone (LH), follicle stimulating hormone (FSH), total testosterone $(T)$, and spermiogram.

Results: During the study period, 33 patients were diagnosed with Klinefelter syndrome (KS+) and 72 were not (KS-). Out of all KS cases, only seven $(21.2 \%)$ were diagnosed before 20 years old and two $(6.1 \%)$ before 10 years old. Age at first consultation (in years) was similar in both groups (KS+ $=31.3 \pm 12.9$ and KS- $=27.6 \pm 12.1$ ), as were ratio of arm span to height and frequency of gynecomastia. However, in KS+ patients, pubic hair was less developed, testicular volume was smaller and testosterone levels were lower, while LH and FSH levels and frequency of azoospermia were higher.

Conclusions: Klinefelter syndrome is both an under and late diagnosed condition. The most important data for diagnosis are testicular volume, hormone levels and presence of azoospermia in spermiogram, especially in puberty and adult life.
\end{abstract}

J Pediatr (Rio J). 2012;88(4):323-7: Klinefelter syndrome, male infertility, delayed puberty, gynecomastia.

1. Residente, Endocrinologia Pediátrica. Departamento de Pediatria, Faculdade de Ciências Médicas (FCM), Universidade Estadual de Campinas (UNICAMP), Campinas, SP.

2. Grupo Interdisciplinar de Estudos da Determinação e Diferenciação do Sexo (GIEDDS), FCM, UNICAMP, Campinas, SP.

3. Graduando, Medicina. FCM, UNICAMP, Campinas, SP.

4. Bióloga. Departamento de Genética Médica, FCM, UNICAMP, Campinas, SP.

5. Médica geneticista clínica. Professora livre-docente. Departamento de Genética Médica, FCM, UNICAMP, Campinas, SP

6. Médica geneticista clínica. Professora titular. Departamento de Genética Médica, FCM, UNICAMP, Campinas, SP.

7. Médico endocrinologista pediátrico. Professor titular. Departamento de Pediatria, FCM, UNICAMP, Campinas, SP

Não foram declarados conflitos de interesse associados à publicação deste artigo.

Como citar este artigo: Tincani BJ, Mascagni BR, Pinto RD, Guaragna-Filho G, Castro CC, Sewaybricker LE, et al. Klinefelter syndrome: an unusual diagnosis in pediatric patients. J Pediatr (Rio J). 2012;88(4):323-7.

Artigo submetido em 12.03.12, aceito em 25.04.12.

http://dx.doi.org/10.2223/JPED.2208 


\section{Introdução}

A síndrome de Klinefelter (SK) é a anomalia de cromossomos sexuais mais comum em homens, com uma prevalência estimada de um em cada 600 homens nascidos vivos $^{1}$. Caracteriza-se citogeneticamente pela presença de um cromossomo X extra $(47, \mathrm{XXY})$, que ocorre em cerca de $90 \%$ dos casos; porém, variantes da SK, como o mosaicismo $(46, X Y / 47, X X Y)$ e outras aneuploidias mais raras $(48, X X X Y$, $48, X X Y Y, 49, X X X X Y)$ já foram descritas ${ }^{2}$.

Os achados clínicos principais, presentes em quase todos os indivíduos com SK, são os testículos pequenos, a azoospermia e o aumento das gonadotrofinas, em especial do follicle stimulating hormone (FSH); porém, outros achados, como ginecomastia, atraso puberal, pilificação pubiana e corporal diminuídas, micropênis, alta estatura, aumento da envergadura em relação à estatura, distúrbios de aprendizado, doenças psiquiátricas, doença venosa periférica, obesidade abdominal, síndrome metabólica, maior risco de doenças autoimunes e câncer, podem ser observados com diferentes frequências de acordo com a população avaliada, a faixa etária incluída e o cariótipo encontrado1,3-10.

Apesar de descrita pela primeira vez há $70 \operatorname{anos}^{11}$, a SK continua sendo uma doença pouco diagnosticada, pois os pacientes procuram pouco os médicos, e os médicos nem sempre estão atentos ao diagnóstico ${ }^{8}$. Por isso, apenas cerca de $25 \%$ de todos os pacientes adultos com SK são diagnosticados; a maioria durante a investigação de infertilidade e/ou hipogonadismo; e menos de $10 \%$ de todos os casos com SK são diagnosticados antes da puberdade ${ }^{1,12}$.

Portanto, o objetivo deste estudo foi identificar dados clínicos e laboratoriais que diferenciam os casos de SK de acordo com a faixa etária.

\section{Casuística e métodos}

Trata-se de um estudo clínico retrospectivo com a inclusão de todos os indivíduos que procuraram o Ambulatório do Grupo Interdisciplinar de Estudos da Determinação e Diferenciação do Sexo no Hospital de Clínicas (HC) da Faculdade de Ciências Médicas (FCM) da Universidade Estadual de Campinas (UNICAMP), no período de janeiro de 1989 a dezembro de 2011, para investigação de SK. Deve-se ressaltar que a consulta neste ambulatório é a única possibilidade de se realizar o cariótipo no HC-UNICAMP quando a suspeita é de SK.

Todos foram avaliados de acordo com a idade na primeira consulta, a queixa clínica principal (infertilidade, ginecomastia, deficiência intelectual e/ou atraso neuromotor, evidências de hipogonadismo, incluindo atraso puberal, desenvolvimento incompleto dos caracteres sexuais secundários, microrquidia e micropênis), relação entre envergadura e altura (E/A), pilificação pubiana por classificação de acordo com Marshall \& Tanner ${ }^{13}$, presença de ginecomastia, volume testicular (medido por orquidômetro de Prader), luteinizing hormone (LH), FSH, testosterona total ( $\mathrm{T}$ ), espermograma e cariótipo. $O$ volume testicular foi calculado pela média aritmética entre o volume dos dois testículos de cada paciente.
Todas as avaliações laboratoriais (LH, FSH, T e espermograma) foram realizadas no Laboratório de Fisiologia do HC-UNICAMP. Em relação ao espermograma, os resultados foram categorizados em normal, oligospermia ou azoospermia. O exame de cariótipo foi realizado em leucócitos de sangue periférico com contagem de no mínimo 32 metáfases no Laboratório de Citogenética do Departamento de Genética Médica da FCM-UNICAMP. A partir do resultado do cariótipo, os pacientes foram divididos em portadores $(\mathrm{SK}+)$ ou não (SK-) da SK.

Os dados avaliados foram comparados entre os casos SK+ e SK- por teste qui-quadrado ou exato de Fisher para as variáveis categóricas (pilificação pubiana, ginecomastia e espermograma) e teste de Mann-Whitney para as variáveis contínuas (idade, E/A, tamanho testicular, LH, FSH e T) com $p<0,05$. O programa utilizado na análise estatística foi o Statistical Package for the Social Sciences (SPSS, Chicago, IL, EUA) versão 16.0.

\section{Resultados}

No total, foram incluídos no estudo 105 casos, 72 com cariótipo normal $(46, X Y), 31$ com cariótipo 47,XXY e dois com cariótipo $46, \mathrm{XY} / 47, \mathrm{XXY}$, sendo, portanto, 72 casos $(68,6 \%)$ sem SK (SK-) e $33(31,4 \%)$ com SK (SK+).

A idade na primeira consulta, no grupo SK-, variou de 1,5 a 67,5 anos (27,6 $\pm 12,1$ anos), e no grupo SK+, de 5,3 a 59,7 anos (31,3 312,9 anos), não havendo diferença estatística entre os grupos $(p=0,13)$. Dos 105 casos, $30(28,6 \%)$ foram avaliados antes dos 20 anos de idade, sendo sete SK+ e 23 SK-; e apenas três antes dos 10 anos de idade, sendo dois $\mathrm{SK}+$ e um SK-. Portanto, entre os três casos de SK+, sete $(21,2 \%)$ foram diagnosticados antes dos 20 anos de idade, e dois $(6,1 \%)$, antes dos 10 anos.

A Tabela 1 mostra a queixa principal na primeira consulta em relação à idade ( $\leq$ e $>20$ anos) e ao diagnóstico $S K+$ e SK-. Independentemente do diagnóstico, a principal queixa acima dos 20 anos foi a de infertilidade (77,5\% no grupo SKe $57,7 \%$ no grupo SK+), enquanto que abaixo de 20 anos, o hipogonadismo e a ginecomastia foram as principais queixas ( $91,3 \%$ no grupo SK- e $71,4 \%$ no grupo $\mathrm{SK}+$ ). A queixa de deficiência mental ou atraso neuromotor só ocorreu abaixo dos 20 anos de idade, independente do diagnóstico ou não de SK; e todos esses pacientes tinham menos de 10 anos de idade. Não se observou diferença estatística entre os grupos SK+ e SK- em relação à queixa principal quando separados por faixa etária ( $\leq 20$ anos: qui-quadrado $(2)=4,29 ; p=0,12$; e $>20$ anos: qui-quadrado $(2)=5,10 ; p=0,08)$.

A Tabela 2 apresenta os dados de estadiamento da pilificação pubiana e da presença ou não de ginecomastia apenas entre os pacientes acima de 10 anos de idade $(n=102 ; 31$ SK+ e 71 SK-) e do espermograma entre os pacientes acima de 18 anos ( $n=79 ; 29$ SK+ e 50 SK-). Observou-se diferença estatisticamente significativa entre os dois grupos no estadiamento da pilificação pubiana (qui-quadrado $=26,22 ; p<0,0001$ ), com o grupo SK+ apresentando menor pilificação pubiana. Também se observou diferença estatisticamente significativa no resultado do espermograma (qui-quadrado $_{(2)}=25,16$; 
Tabela 1 - Motivo da consulta em relação à idade e ao diagnóstico ou não de síndrome de Klinefelter

\begin{tabular}{|c|c|c|c|c|}
\hline \multirow[b]{2}{*}{ Motivo da consulta } & \multicolumn{2}{|c|}{ SK- $(n=72)$} & \multicolumn{2}{|c|}{ SK+ $(n=33)$} \\
\hline & $\leq 20$ anos & $>20$ anos & $\leq 20$ anos & $>20$ anos \\
\hline Infertilidade & 0 & 38 & 0 & 15 \\
\hline Hipogonadismo & 14 & 8 & 2 & 5 \\
\hline Ginecomastia & 8 & 3 & 3 & 6 \\
\hline Deficiência mental & 1 & 0 & 2 & 0 \\
\hline Total & 23 & 49 & 7 & 26 \\
\hline
\end{tabular}

SK- = síndrome de Klinefelter ausente; SK+ = síndrome de Klinefelter presente.

$\mathrm{p}<0,0001)$, com o grupo SK+ apresentando apenas azoospermia. Porém, não se observou diferença estatisticamente significativa na presença de ginecomastia (qui-quadrado = 2,$68 ; p=0,10$ ) entre os dois grupos.

A Tabela 3 mostra os dados de E/A de todos os casos e do volume testicular médio, LH, FSH e T apenas daqueles acima de 14 anos ( $n=92 ; 31$ SK+ e 61 SK-). Não se observou diferença estatisticamente significativa entre os dois grupos na $E / A(p=0,98)$; porém, se observou diferença estatisticamente significativa no volume testicular médio $(p=0,001)$, no LH $(p=0,001)$, no FSH $(p=0,001)$ e na T $(p=0,01)$, com os valores de volume testicular médio e $T$ menores e de FSH e LH maiores no grupo SK+.

\section{Discussão}

Os dados deste estudo mostram que a SK ainda é pouco e tardiamente diagnosticada em nosso meio. O HC-UNICAMP atende a população de uma região com mais de 5 milhões de habitantes, e para esse volume de atendimento deveríamos ter registrado muito mais que 33 diagnósticos de SK em 23 anos (cerca de três casos diagnosticados a cada 2 anos), indicando que ou os pacientes procuram pouco o serviço médico, ou os médicos pensam pouco nesse diagnóstico. Apesar de aparentemente pequena, a casuística do presente estudo é parecida com a maioria dos estudos recentes ${ }^{1,3,8,14}$, onde a maior foi de $98 \operatorname{casos}^{10}$.

Em relação à idade ao diagnóstico, dos 105 casos incluídos no estudo, apenas 30 (28,6\%) foram avaliados antes dos 20 anos de idade, sendo sete destes (23,3\%) com diagnóstico de SK e apenas três (2,9\%) avaliados abaixo dos 10 anos de idade, sendo dois destes com diagnóstico de SK. Portanto, os dados deste estudo confirmam que raramente o diagnóstico da SK é feito antes dos 20 anos de idade, especialmente antes dos 10 anos de idade. Dos 75 casos avaliados após os 20 anos de idade, o diagnóstico da SK ocorreu com maior frequência, na proporção 1:2, ou seja, a cada três casos, em um foi feito o diagnóstico da SK. Resultados semelhantes foram observados por Abramsky \& Chapple ${ }^{12}$, ao verificarem que $26 \%$ do número esperado de casos de SK em North Thames, no Reino Unido, entre 1990 e 1993, foram diagnosticados após o nascimento,
Tabela 2 - Pilificação pubiana, ginecomastia e espermograma em relação ao diagnóstico ou não de síndrome de Klinefelter

\begin{tabular}{lccc}
\hline Variável & SK- & SK+ & p \\
\hline $\begin{array}{l}\text { Pilificação } \\
1 \text { ou } 2\end{array}$ & 5 & 16 & $<0,0001$ \\
$\quad 3$ a 5 & 66 & 15 & \\
Ginecomastia & & & 0,10 \\
$\quad+$ & 31 & 19 & \\
$\quad-$ & 40 & 12 & \\
Espermograma & & & \\
$\quad$ Normal & 7 & 0 & \\
$\quad$ Oligospermia & 21 & 0 & \\
Azoospermia & 22 & 29 & \\
\end{tabular}

SK- = síndrome de Klinefelter ausente; SK+ = síndrome de Klinefelter presente.

1 a 5 = estadiamento de acordo com Marshall \& Tanner ${ }^{13} ;$ pilificação pubiana e ginecomastia foram avaliadas apenas nos pacientes com idade acima de 10 anos, e o espermograma, apenas naqueles acima de 18 anos.

Tabela 3 - Relação envergadura/altura, volume testicular médio, hormônio luteinizante, hormônio folículo-estimulante e testosterona total em relação ao diagnóstico ou não de síndrome de Klinefelter

\begin{tabular}{|c|c|c|c|}
\hline Variável & $\frac{\text { SK- }}{(M \pm D P)}$ & $\frac{S K+}{(M \pm D P)}$ & p \\
\hline Envergadura/altura & $1,02 \pm 0,03$ & $1,01 \pm 0,03$ & 0,98 \\
\hline Volume testicular médio $(\mathrm{mL})$ & $9,5 \pm 6,5$ & $3,5 \pm 2,5$ & 0,001 \\
\hline $\mathrm{LH}(\mathrm{U} / \mathrm{L})$ & $7,5 \pm 8,4$ & $19,6 \pm 8,8$ & 0,001 \\
\hline $\mathrm{FSH}(\mathrm{U} / \mathrm{L})$ & $14,9 \pm 7,4$ & $43,8 \pm 25,4$ & 0,001 \\
\hline $\mathrm{T}(\mathrm{ng} / \mathrm{mL})$ & $8,7 \pm 7,3$ & $4,3 \pm 2,9$ & 0,01 \\
\hline
\end{tabular}

FSH = hormônio folículo-estimulante; LH = hormônio luteinizante; $\mathrm{M} \pm \mathrm{DP}=$ média \pm desvio padrão; SK- = síndrome de Klinefelter ausente; $\mathrm{SK}+=$ síndrome de Klinefelter presente; $\mathrm{T}$ = testosterona total. 
sendo $28 \%$ destes diagnosticados antes dos 20 anos de idade e apenas $4 \%$ antes dos 10 anos de idade; dados semelhantes aos encontrados por Bojesen et al. ${ }^{1}$ e pelo presente estudo.

Todos os indivíduos avaliados abaixo dos 10 anos de idade foram encaminhados por deficiência intelectual e/ou atraso do desenvolvimento neuromotor. Déficits cognitivos específicos na linguagem e na função executiva são parte do fenótipo da $\mathrm{SK}^{15}$. Apesar de muitos estudos relatarem apenas um leve comprometimento cognitivo global (atraso leve no desenvolvimento motor e da linguagem), a SK tem sido identificada em $0,4 \%$ dos meninos em programas de educação especial e em $1,2 \%$ dos pacientes com retardo intelectual de causa indeterminada 16,17 ; em geral, referidos inicialmente para a investigação de síndrome do $\mathrm{X}$ frágili18, principal causa hereditária de retardo mental, mais comum no sexo masculino e com manifestações pouco expressivas antes da puberdade, exceto pelo comprometimento intelectual e as alterações de comportamento. Na puberdade e após, a principal característica clínica que diferencia SK de síndrome do $X$ frágil é o volume testicular, pequeno na primeira e grande na segunda.

Acima dos 20 anos de idade, a principal queixa na suspeita diagnóstica foi a de infertilidade, enquanto abaixo de 20 anos, porém já na fase de puberdade, acima dos 10 anos de idade, o hipogonadismo e a ginecomastia foram as principais queixas. A pilificação pubiana reduzida e o menor volume testicular médio foram significativamente mais frequentes no grupo SK+, o mesmo não ocorrendo com a ginecomastia e o predomínio da envergadura sobre a estatura. A ginecomastia é um achado frequente da SK (50 a 88\%) e da puberdade normal masculina (60 a 70\%), havendo portanto, sobreposição dos diagnósticos e, por isso, não sendo um achado de importância significativa no diagnóstico da SK na puberdade ${ }^{19}$. $A$ redução da pilificação pubiana e a relação E/A aumentada estão relacionadas ao hipogonadismo, que frequentemente não é suspeitado na puberdade, pois a maioria dos pacientes com SK inicia puberdade na época normal ${ }^{19}$.

Em relação aos exames laboratoriais, o LH, o FSH, a Te a frequência de azoospermia no espermograma foram significativamente maiores no grupo SK+. Do ponto de vista hormonal, pacientes com SK apresentam valores pré-puberais normais de T, LH, FSH, inibina B e hormônio antimülleriano, com resposta normal da T ao teste de estímulo com gonadotrofina coriônica humana $9,10,20,21$. Durante a puberdade, após um aumento normal da T, esta pode, em alguns casos, se manter em níveis normais baixos para o estadio de maturação sexual, porém com condições de progredir e determinar o desenvolvimento satisfatório das características sexuais secundárias 9,10,20,21. A partir da metade da puberdade, em geral, os pacientes com SK já começam a apresentar um aumento gradual do FSH (refletindo a disfunção dos túbulos seminíferos e das células de Sertoli) e do LH (refletindo a disfunção das células de Leydig), sendo que o aumento do FSH em geral precede (cerca de 1 ano antes) e é mais intenso do que o de LH; tais dados podem, a partir deste momento, auxiliar na suspeita diagnóstica, como verificado no presente estudo. No entanto, o principal dado clínico na faixa etária puberal para justificar a determinação laboratorial de LH e FSH e, se aumentados, a realização do cariótipo é a desproporção do volume testicular para o estádio de maturação sexual. Kamischke et al. ${ }^{3}$ mostraram que a medida média do volume dos testículos foi o parâmetro de melhor sensibilidade para o diagnóstico de SK. Esse estudo mostrou que a média do volume bitesticular por ultrassonografia foi de 4,7 mL nos casos SK+ e 13,7 mL nos SK-, dados semelhantes aos encontrados no presente estudo por avaliação clínica.

$\mathrm{Na}$ idade adulta, os principais dados que motivam a investigação da SK são o hipogonadismo (clínico) e hipergonadotrófico (laboratorial) e a infertilidade ${ }^{19}$. A SK é a causa genética mais frequente de infertilidade masculina, ocorrendo em $11 \%$ dos homens azoospérmicos e $4 \%$ dos homens inférteis $^{22}$. No presente estudo, esses dados se confirmaram, sendo a infertilidade e o hipogonadismo as principais queixas após os 20 anos de idade, e todos os pacientes SK+ acima de 18 eram azoospérmicos.

A maioria dos achados clínicos e laboratoriais presentes na SKé decorrente do processo degenerativo testicular presente durante toda a vida. No feto e no neonato, ocorre a diminuição do número de células germinativas e um aumento da proporção de túbulos seminíferos sem células germinativas, continuando com a redução da luz dos túbulos seminíferos, maior diminuição do número de espermatogônias na criança e aceleração de todo esse processo na puberdade, finalizando no adulto com extensa fibrose e hialinização dos túbulos seminíferos, ausência de espermatogônias, predomínio de células de Sertoli imaturas e hiperplasia das células de Leydig e do interstício ${ }^{23}$.

É importante salientar que entre os 33 indivíduos com SK do presente estudo, 31 apresentavam cariótipo 47, XXY e dois $46, X Y / 47, X X Y$, pois, em geral, os pacientes com mosaicismo apresentam fenótipo menos grave 24,25 , porém sem diferença na morbidade e mortalidade ${ }^{14}$, enquanto que os pacientes com cariótipo 48, XXXY e 49,XXXXY apresentam fenótipo mais grave, em especial quanto ao comprometimento intelectual e na associação com malformações ${ }^{26}$, porém também sem aparente aumento da mortalidade 5,14 .

Diante disso, podemos concluir que o diagnóstico da SK em nosso meio não está sendo feito com a frequência esperada e na época adequada, e as principais consequências poderão ser a falta de tratamento do déficit motor e cognitivo na idade escolar, do atraso puberal ou da puberdade incompleta na adolescência ou no adulto jovem e da infertilidade na vida adulta. É oportuno que os profissionais de saúde, em especial na faixa etária pediátrica, como pediatras em geral, neurologistas e endocrinologistas pediátricos e fonoaudiólogos, fiquem mais atentos para o diagnóstico da SK.

\section{Referências}

1. Bojesen A, Juul S, Gravholt CH. Prenatal and postnatal prevalence of Klinefelter syndrome: a national registry study. J Clin Endocrinol Metab. 2003;88:622-6.

2. Lanfranco F, Kamischke A, Zitzmann M, Nieschlag E. Klinefelter's syndrome. Lancet. 2004;364:273-83. 
3. Kamischke A, Baumgardt A, Horst J, Nieschlag E. Clinical and diagnostic features of patients with suspected Klinefelter syndrome. J Androl. 2003;24:41-8.

4. Swerdlow AJ, Higgins $C D$, Schoemaker $M J$, Wright $A F$, Jacobs PA; United Kingdom Clinical Cytogenetics Group. Mortality in patients with Klinefelter syndrome in Britain: a cohort study. J Clin Endocrinol Metab. 2005;90:6516-22.

5. Swerdlow AJ, Schoemaker MJ, Higgins CD, Wright AF, Jacobs PA; UK Clinical Cytogenetics Group. Cancer incidence and mortality in men with Klinefelter syndrome: a cohort study. J Natl Cancer Inst. 2005; 97:1204-10.

6. Bojesen A, Juul S, Birkebaek NH, Gravholt CH. Morbidity in Klinefelter syndrome: a Danish register study based on hospital discharge diagnoses. J Clin Endocrinol Metab. 2006;91:1254-60.

7. Bojesen A, Gravholt CH. Klinefelter syndrome in clinical practice. Nat Clin Pract Urol. 2007;4:192-204.

8. Bojesen A, Gravholt CH. Morbidity and mortality in Klinefelter syndrome (47,XXY). Acta Paediatr. 2011;100:807-13.

9. Wikström AM, Dunkel L. Klinefelter syndrome. Best Pract Res Clin Endocrinol Metab. 2011;25:239-50.

10. Pacenza N, Pasqualini T, Gottlieb S, Knoblovits P, Costanzo PR, Stewart Usher J, et al. Clinical presentation of Klinefelter's syndrome: differences according to age. Int J Endocrinol. 2012;2012:324835.

11. Klinefelter HF Jr, Reifenstein EC Jr, Albright F. Syndrome characterized by gynaecomastia, aspermatogenesis without a-Leydigism, and increase secretion of follicule-stimulating hormone. J Clin Endocrinol Metab. 1942;2:615-27.

12. Abramsky L, Chapple J. 47,XXY (Klinefelter syndrome) and 47,XYY: estimated rates of and indication for postnatal diagnosis with implications for prenatal counselling. Prenat Diagn. 1997; 17:363-8.

13. Marshall WA, Tanner JM. Variations in the pattern of pubertal changes in boys. Arch Dis Child. 1970;45:13-23.

14. Bojesen A, Juul S, Birkebaek N, Gravholt CH. Increased mortality in Klinefelter syndrome. J Clin Endocrinol Metab. 2004;89:3830-4.

15. Wattendorf DJ, Muenke M. Klinefelter syndrome. Am Fam Physician. 2005; 72:2259-62.

16. Fales CL, Knowlton BJ, Holyoak KJ, Geschwind DH, Swerdloff RS, Gonzalo IG. Working memory and relational reasoning in Klinefelter syndrome. J Int Neuropsychol Soc. 2003;9:839-46.
17. Khalifa MM, Struthers JL. Klinefelter syndrome is a common cause for mental retardation of unknown etiology among prepubertal males. Clin Genet. 2002;61:49-53.

18. Youings SA, Murray A, Dennis N, Ennis S, Lewis C, McKechnie N, et al. FRAXA and FRAXE: the results of a five year survey. J Med Genet. 2000;37:415-21.

19. Radicioni AF, Ferlin A, Balercia G, Pasquali D, Vignozzi L, Maggi M, et al. Consensus statement on diagnosis and clinical management of Klinefelter syndrome. J Endocrinol Invest. 2010;33:839-50.

20. Salbenblatt JA, Bender BG, Puck MH, Robinson A, Faiman C, Winter JS. Pituitary-gonadal function in Klinefelter syndrome before and during puberty. Pediatr Res. 1985;19:82-6.

21. Topper E, Dickerman Z, Prager-Lewin R, Kaufman H, Maimon Z, Laron Z. Puberty in 24 patients with Klinefelter syndrome. Eur J Pediatr. 1982;139:8-12.

22. Van Assche $E$, Bonduelle $M$, Tournaye $H$, Joris $H$, Verheyen $G$, Devroey $P$, et al. Cytogenetics of infertile men. Hum Reprod. 1996;11:1-24; discussion 25-6.

23. Wikström AM, Dunkel L. Testicular function in Klinefelter syndrome. Horm Res. 2008;69:317-26.

24. Sarkar R, Marimuthu KM. Association between the degree of mosaicism and the severity of syndrome in Turner mosaics and Klinefelter mosaics. Clin Genet. 1983;24:420-8.

25. Smyth CM, Bremner WJ. Klinefelter syndrome. Arch Intern Med. 1998; $158: 1309-14$.

26. Visootsak J, Aylstock M, Graham JM Jr. Klinefelter syndrome and its variants: an update and review for the primary pediatrician. Clin Pediatr (Phila). 2001;40:639-51.

\author{
Correspondência: \\ Gil Guerra-Júnior \\ Departamento de Pediatria - FCM - UNICAMP \\ Cidade Universitária "Zeferino Vaz", s/n \\ CEP 13081-970 - Campinas, SP \\ Tel.: (19) 3521.7353 \\ Fax: (19) 3521.7322 \\ E-mail: gilguer@fcm.unicamp.br
}

